\title{
Article \\ Synthesis, Antibacterial and Anthelmintic Activity of Novel 3-(3-Pyridyl)-oxazolidinone-5-methyl Ester Derivatives
}

\author{
Bo Jin ${ }^{1}{ }^{\circledR}$, Jia-yi Chen ${ }^{1}$, Zun-lai Sheng ${ }^{1,2}$, Meng-qing Sun ${ }^{1,2}$ and Hong-liang Yang ${ }^{1,2, *}$ \\ 1 Department of Veterinary Medicine, Northeast Agricultural University, Harbin 150030, China; \\ cnborgin@163.com (B.J.); bear2320581078@163.com (J.-y.C.); shengzunlai@neau.edu.cn (Z.-1.S.); \\ smq1536578652@163.com (M.-q.S.) \\ 2 Heilongiiang Key Laboratory for Animal Disease Control and Pharmaceutical Development, \\ Northeast Agricultural University, Harbin 150030, China \\ * Correspondence: hongl_yang@126.com
}

Citation: Jin, B.; Chen, J.-y.; Sheng, Z.-1.; Sun, M.-q.; Yang, H.-1. Synthesis, Antibacterial and Anthelmintic Activity of Novel 3-(3-Pyridyl)oxazolidinone-5-methyl Ester Derivatives. Molecules 2022, 27, 1103. https://doi.org/10.3390/ molecules 27031103

Academic Editor: Victor Mamane

Received: 25 December 2021

Accepted: 3 February 2022

Published: 7 February 2022

Publisher's Note: MDPI stays neutral with regard to jurisdictional claims in published maps and institutional affiliations.

Copyright: (C) 2022 by the authors. Licensee MDPI, Basel, Switzerland. This article is an open access article distributed under the terms and conditions of the Creative Commons Attribution (CC BY) license (https:// creativecommons.org/licenses/by/ $4.0 /)$.

\begin{abstract}
In this study, a series of 3-(3-pyridyl)-oxazolidone-5-methyl ester derivatives was synthesized and characterized by ${ }^{1} \mathrm{H}$ NMR, ${ }^{13} \mathrm{C}$ NMR, and LC-MS. The conducted screening antibacterial studies of the new 3-(3-pyridyl)-oxazolidone-5-methyl ester derivatives established that the methyl sulfonic acid esters have broad activity spectrum towards Staphylococcus aureus, Streptococcus pneumoniae, Bacillus subtilis and Staphylococcus epidermidis. Among them, compound 12e has the most potent activity, with an MIC of $16 \mu \mathrm{g} / \mathrm{mL}$ against B.subtilis, and could reduce the instantaneous growth rate of bacteria. Furthermore, molecular docking studies were also simulated for compound 12e to predict the specific binding mode of this compound. In addition, anthelmintic activity of these compounds was also evaluated against adult Indian earthworms (Pheretima posthuman). The results showed that compound $\mathbf{1 1 b}$ had the best effect. These results above can provide experimental reference for the development of novel antibacterial and anthelmintic drugs.
\end{abstract}

Keywords: pyridinyl-oxazolidinone derivatives; synthesis; antibacterial activity; molecular docking; anthelmintic activity

\section{Introduction}

With bacterial resistance comes a grave threat to global public health security. Owing to the rapid growth of clinical drug-resistant bacteria, the number of effective anti-infective drugs has declined, and patient mortality is increasing [1-5]. It is estimated that about 10 million people all over the world will die from drug-resistant bacterial infections, which will become the leading cause of human disease death, and lead to the loss of global GDP of US $\$ 100$ trillion by 2050 [6]. Therefore, how to effectively deal with the bacterial resistance crisis is a challenging question. Many new therapies, such as those utilizing nanoparticles, phages, and protic ionic liquids, are being developed [7-11]. However, these technologies are still immature and have not been effectively used clinically [12-14]. At present, chemosynthesized antibacterial agents still play an indispensable role in clinical practice. Therefore, for pharmaceutical chemists all over the world, the discovery of novel active antibacterial compounds is an urgent mission and the direction of continuous efforts.

Linezolid, an oxazolidinone antibacterial agent, has been widely used in the treatment of infection caused by multi-drug-resistant Gram-positive bacteria since it was approved for clinical use in 2000. However, with clinical use gradually increasing, many linezolidresistant strains have also been reported [15-18]. To find novel, safe and effective antibacterial drugs, there have been many studies in recent years on the structural modification of linezolid [19-25]. Among them, many have focused on the modification of five-position side chains of oxazolidine and the morpholine ring, while a few have explored the benzene ring of linezolid. In addition, anthelmintic properties of linezolid derivatives have also been found [26]. 
The pyridine heterocyclic ring has been widely used in the medicine field because of its distinctive aromatic and electronegative properties [27-31]. In our previous studies, a series of pyridine heterocyclic derivatives were evaluated to have good biological activities [32-34]. Among them, compound 1 showed good antibacterial and anthelmintic activities in vitro. Based on previous research and theory [35], here we replaced the benzene ring with pyridine and a nitrogen atom with an oxygen atom at the 5-position side chain of the oxazolidone ring. A series of 3-(3-pyridyl)-oxazolidinone-5-methyl ester derivatives were designed and synthesized (Figure 1), and their antibacterial and anthelmintic effects in vitro were evaluated.

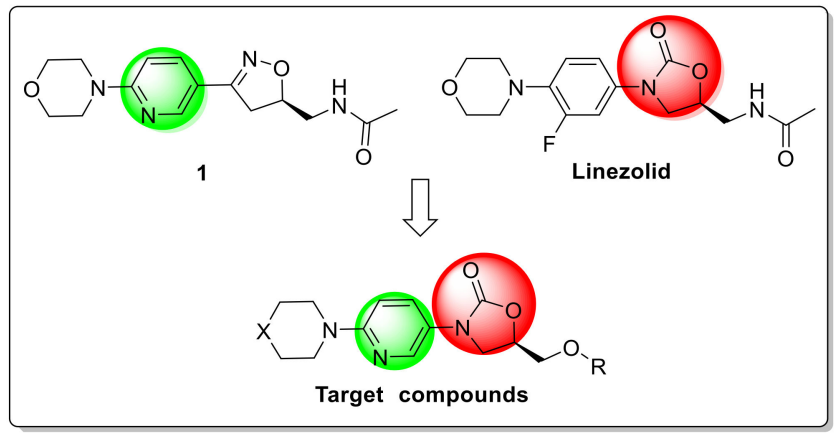

Figure 1. Design strategy of 3-(3-pyridyl)-oxazolidone-5-methyl ester derivatives.

\section{Results and Discussion}

\subsection{Chemistry}

The general synthetic route for the target compounds is illustrated in Scheme 1. The commercially available 2-chloro-5-nitropyridine was reacted with morpholine to produce intermediate 2 . Then, the nitro group was reduced to prepare amine 4 . After the introduction of the $\mathrm{Cbz}$ group, intermediate 6 was cyclized with (R)-(-)-butylglycidyl ester under the condition of $-78{ }^{\circ} \mathrm{C}$ and n-butyllithium (n-BuLi) to produce oxazolidinone intermediate 9. Finally, ester derivatives 11a-i were prepared with intermediate 9 and different acids, sulfonyl chlorides and isocyanates. Compounds $\mathbf{1 2 a}-\mathbf{h}$ were obtained by similar methods.
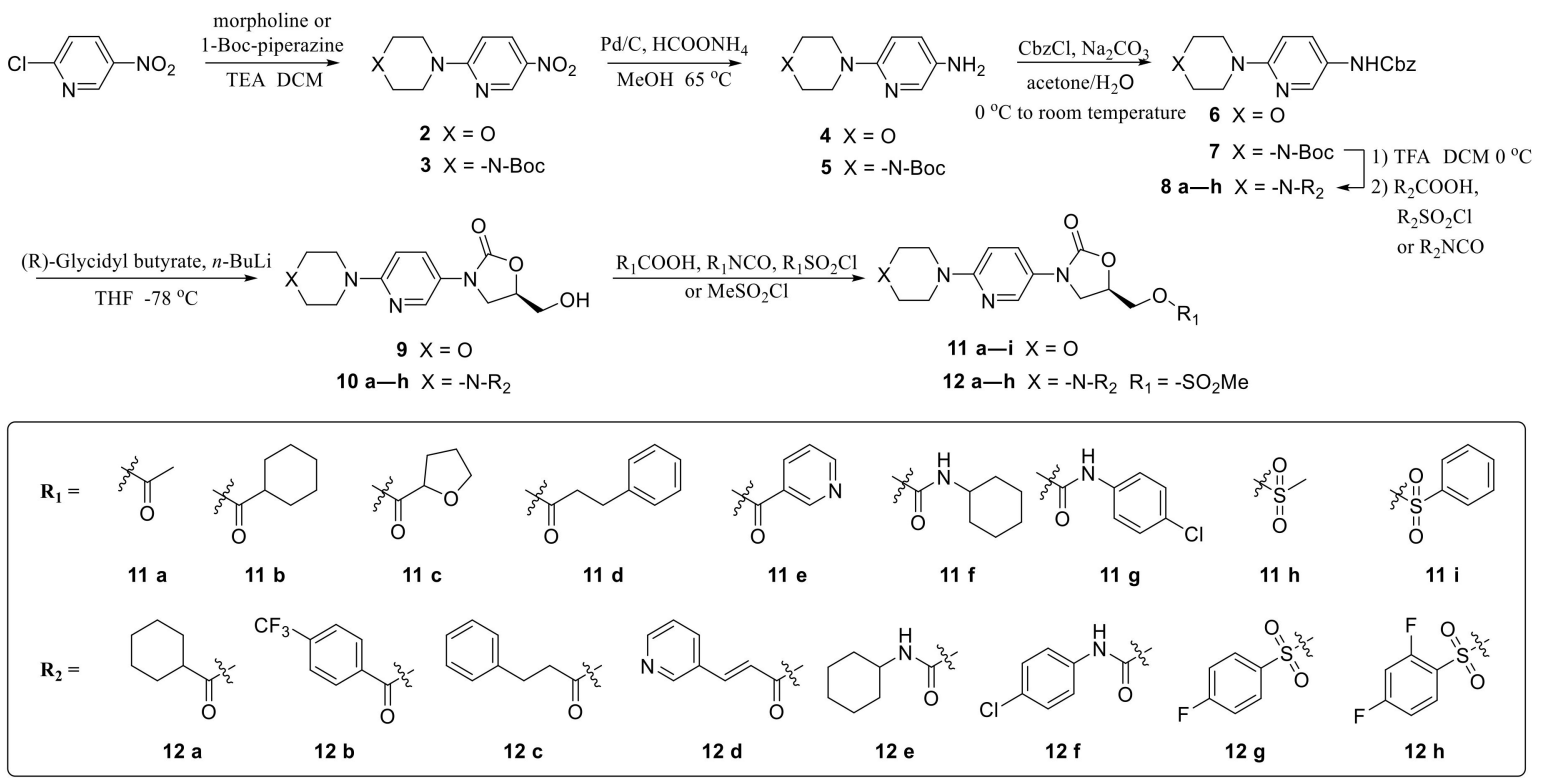

Scheme 1. Synthesis route of the target compounds. 


\subsection{Antibacterial Activity Assay}

\subsubsection{Screening of Antibacterial Activity}

All twelve synthesized compounds were tested against four Gram-positive strains, including S. aureus (ATCC25923), S. pneumoniae (ATCC49619), B. subtilis (BNCC109047) and S. epidermidis (BNCC186652) by broth dilution method with linezolid as the standard drug, and the MIC values are listed in Table 1.

Table 1. In vitro antibacterial activity of target derivatives (MIC: $\mu \mathrm{g} / \mathrm{mL}$ ).

\begin{tabular}{|c|c|c|c|c|}
\hline \multirow[b]{2}{*}{ Compound } & \multicolumn{4}{|c|}{ Antimicrobial Screening MIC ( $\mu \mathrm{g} / \mathrm{mL})$} \\
\hline & $\begin{array}{c}\text { S. Aureus } \\
\text { Atcc } 25923\end{array}$ & $\begin{array}{l}\text { S. Pneumoniae } \\
\text { Atcc } 49619\end{array}$ & $\begin{array}{c}\text { B. Subtilis } \\
\text { Bncc } 109047\end{array}$ & $\begin{array}{c}\text { S. Epidermidis } \\
\text { Bncc } 186652\end{array}$ \\
\hline $11 a$ & 128 & $>256$ & 256 & $>256$ \\
\hline $11 b$ & 128 & $>256$ & 128 & $>256$ \\
\hline 11c & $>256$ & $>256$ & 128 & $>256$ \\
\hline 11d & $>256$ & $>256$ & 256 & $>256$ \\
\hline 11e & $>256$ & $>256$ & $>256$ & $>256$ \\
\hline $11 f$ & 256 & 128 & 128 & 128 \\
\hline $11 \mathrm{~g}$ & $>256$ & 256 & $>256$ & 128 \\
\hline 11h & 128 & 128 & 64 & 128 \\
\hline $11 \mathbf{i}$ & $>256$ & 256 & $>256$ & $>256$ \\
\hline $12 a$ & 32 & 32 & 32 & 16 \\
\hline $12 b$ & 128 & 128 & 128 & 128 \\
\hline $12 c$ & 64 & 32 & 32 & 32 \\
\hline $12 \mathrm{~d}$ & 32 & 64 & 64 & 64 \\
\hline $12 \mathrm{e}$ & 32 & 32 & 16 & 32 \\
\hline $12 f$ & 128 & 128 & 64 & 64 \\
\hline $12 \mathrm{~g}$ & 128 & 32 & 32 & 128 \\
\hline $12 \mathrm{~h}$ & 128 & 128 & 128 & 128 \\
\hline Linezolid & 2 & 2 & 2 & 2 \\
\hline
\end{tabular}

MIC: minimal inhibit concentration.

Initially, keeping the morpholine ring of linezolid in our structure, compounds 11a-i were synthesized and evaluated for antibacterial activity against four gram-positive strains. Unfortunately, the activity of most compounds was generally weak, with only compounds 11f and 11h showing a broad-spectrum effect.

Therefore, while maintaining the methyl sulfonyl group on the C-5 side chain, compounds $\mathbf{1 2} \mathbf{a}-\mathbf{h}$ were synthesized by replacing the morpholine ring with a piperazine ring and modifying the side chain further. Their activity was significantly improved and they all had broad-spectrum antibacterial action. Among these compounds, compounds 12a and 12e had the best antibacterial activity, with the lowest MIC values reaching $16 \mu \mathrm{g} / \mathrm{mL}$, indicating that the flexible substituents linked with piperazine had stronger antibacterial activity. The activity of $\mathbf{1 2 c}$ and $\mathbf{1 2 d}$ was slightly weaker than that of $\mathbf{1 2 a}$ and $\mathbf{1 2 e}$, probably due to the presence of long-chain substituents, which were less binding than flexible rings, but their long chains were morphologically consistent with the target. Conversely, rigid rings, such as $\mathbf{1 2 b}$, reduced antibacterial activity. Compared with our previous work, these compounds still retain antibacterial activity after changing the core structure to oxazolidinone [34]. Here the antibacterial activity of the synthesized compounds was not as good as linezolid, which may be due to its reduced affinity with the target caused by the structural changes. In addition, a bacterial inhibitory zone test of $\mathbf{1 2} \mathbf{e}$ against $S$. aureus was provided to visually observe bacteriostatic effects of 12e (Figure 2). It can be seen from the figure that the bacteriostatic zone increased with the increase of drug concentration, and the diameter of the zone was similar to that of linezolid at a concentration of $20 \times$ MIC. 

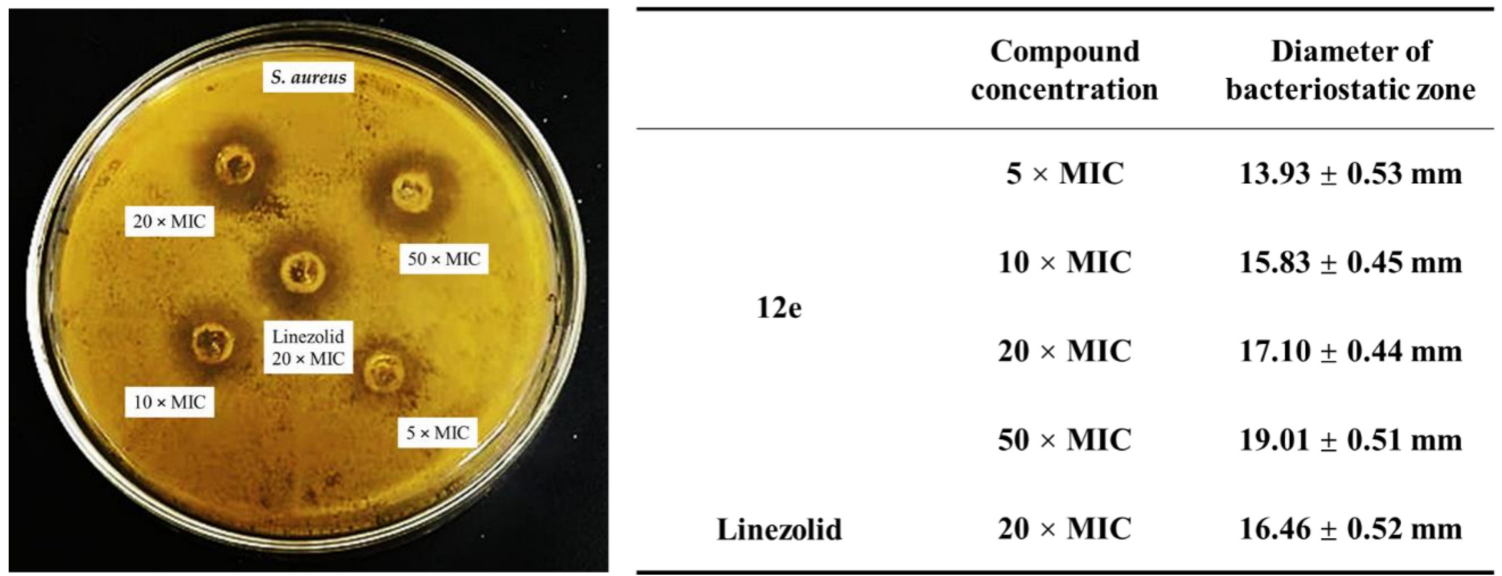

Figure 2. Bacteriostatic zone of $\mathbf{1 2} \mathbf{e}$ against $S$. aureus at different concentrations. (MIC: minimal inhibit concentration).

\subsubsection{Bacterial Growth Kinetic Study}

The growth curve of bacteria directly shows the process and rule of the change of bacterial concentration with time, while the growth inhibition curve of bacteria can directly reflect the influence of drugs on the growth process of bacteria [36]. The growth inhibition curve of compound 12e against Bacillus subtilis is shown in Figure 3.

\section{Bacillus subtilis}

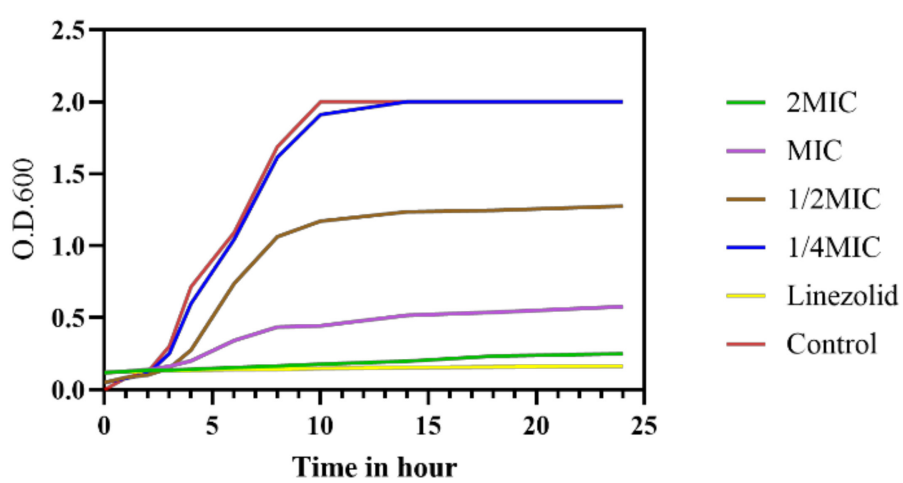

Figure 3. Inhibition of the growth of Bacillus subtilis by different concentrations of compound 12e. (MIC: minimal inhibit concentration; O. D.: optical density.)

Without treatment (red) or the presence of $1 / 4 \mathrm{MIC}$ (blue) of 12e, the bacteria increased sharply in the log phase $(2-10 \mathrm{~h})$. With the increase in concentration, the lag phase was prolonged and the duration of the logarithmic phase was shortened. The significantly change was that the decreased instantaneous growth rate led to the plateau phase concentration reduction, which was manifested by the difference of the final turbidity of bacterial liquid. Until $2 \times$ MIC of 12e (green) and linezolid (yellow), there was no obvious growth of bacteria.

These findings revealed that 12e was an excellent bactericidal agent in a concentrationdependent manner, and mainly disturbed the instantaneous growth rate of bacteria in the logarithmic phase.

\subsection{Binding Mode Study}

To explore the mechanism of the active compound 12e, Auto-Dock software was used to perform docking analysis with the 50S ribosomal subunit (PDB ID: 3CPW).

The docking result from Figure 4 demonstrated that 12e was inclined to locate in a long and narrow channel with a fully extended state. The five-position side chain of the 
oxazolidine ring was binding to a relatively small cavity (yellow), which could only accommodate a small group. This might be the reason for the moderate activity of compound 11h. In addition, it was discovered that the $4^{\prime}$-position side chain of the piperazine ring extended into a deep cavity (red), which could accommodate bigger structural groups after containing the flexible cyclohexyl group. Presumably, compounds $\mathbf{1 2 a}-\mathbf{h}$ had better antibacterial effect than 11a-i, because they were suitable for the narrow cavity (yellow), and occupied the larger and deeper region (red) at the same time, making them more closely combined with the target.

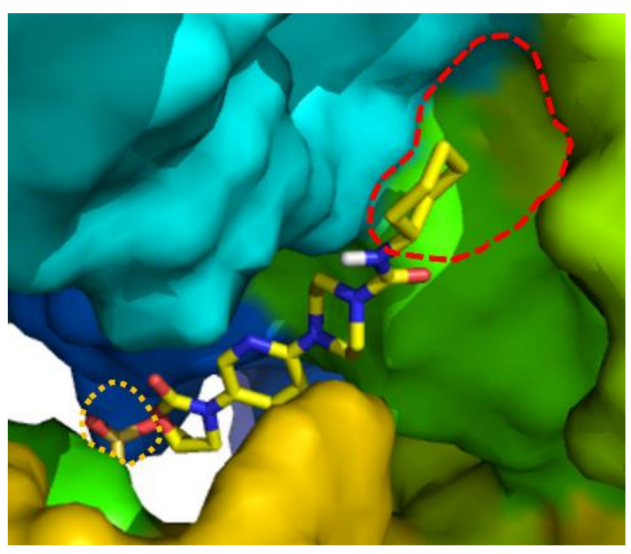

Figure 4. Binding pocket of compound $12 \mathrm{~b}$ with the $50 \mathrm{~S}$ ribosomal subunit.

The specific mechanism of action is shown in Figure 5; the oxygen atoms of the sulfonyl group formed two hydrogen bonds with A2473. It followed that the sulfonyl group presumedly had better ability to form hydrogen bonds than the carbonyl group, which explained why $\mathbf{1 1 h}$ had the best antibacterial activity among 11a-i. In addition, the piperazine-linked urea group of $\mathbf{1 2 e}$ extended into the deep cavity forming a hydrogen bond with G2283, making 12b more effective.

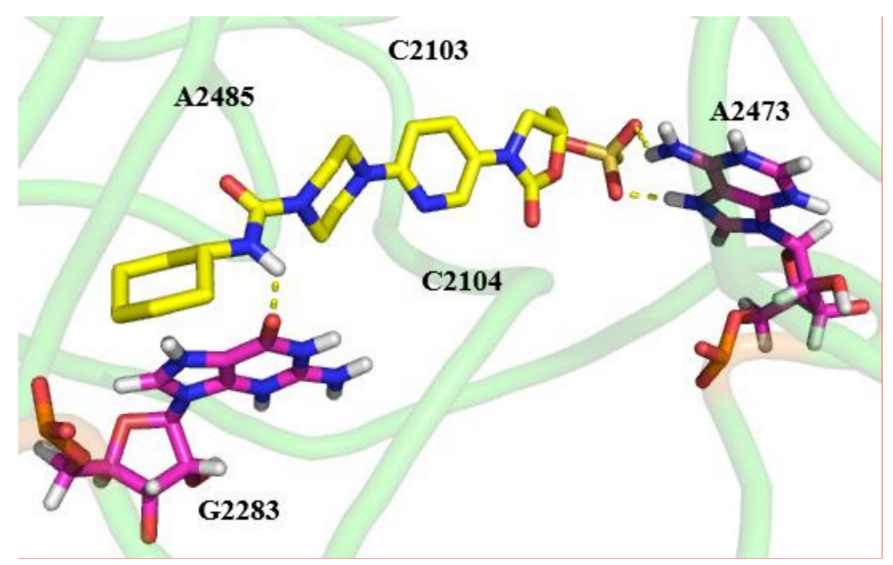

Figure 5. Binding mode of compound 12e to the ribosomal subunit $50 \mathrm{~S}$.

\subsection{Anthelmintic Activity Assay}

Anthelmintic activity assay of 17 target compounds was performed using adult Indian earthworm (Pheretima posthuman) with albendazole as the positive standard drug. Efficacy was assessed by counting the paralysis and death time, and the results are shown in Table 2. 
Table 2. Anthelmintic Activities of Target Compounds.

\begin{tabular}{ccc}
\hline Compound & Mean Paralysis Time/Min & Mean Death Time/Min \\
\hline $\mathbf{1 1 a}$ & $11.0 \pm 1.8$ & $35.9 \pm 5.2$ \\
$\mathbf{1 1 b}$ & $3.6 \pm 0.2$ & $8.1 \pm 0.8$ \\
$\mathbf{1 1} \mathbf{c}$ & $28.3 \pm 5.8$ & $>60$ \\
$\mathbf{1 1 d}$ & $8.6 \pm 1.4$ & $35.6 \pm 6.0$ \\
$\mathbf{1 1}$ & $13.0 \pm 0.4$ & $>60$ \\
$\mathbf{1 1 f}$ & $11.6 \pm 1.0$ & $>60$ \\
$\mathbf{1 1 g}$ & $13.7 \pm 1.7$ & $52.1 \pm 7.0$ \\
$\mathbf{1 1 h}$ & $14.8 \pm 0.9$ & $>60$ \\
$\mathbf{1 1}$ & $18.8 \pm 2.7$ & $>60$ \\
$\mathbf{1 2} \mathbf{a}$ & $17.4 \pm 1.7$ & $55.2 \pm 2.9$ \\
$\mathbf{1 2 b}$ & $22.6 \pm 4.3$ & $>60$ \\
$\mathbf{1 2} \mathbf{c}$ & $19.5 \pm 2.2$ & $50.3 \pm 1.9$ \\
$\mathbf{1 2 d}$ & $18.3 \pm 2.1$ & $55.2 \pm 5.0$ \\
$\mathbf{1 2}$ & $33.1 \pm 2.3$ & $>60$ \\
$\mathbf{1 2 f}$ & $35.8 \pm 2.3$ & $>60$ \\
$\mathbf{1 2} \mathbf{g}$ & $32.6 \pm 3.6$ & $>60$ \\
$\mathbf{1 2 h}$ & $15.6 \pm 1.7$ & $>60$ \\
Albendazole & $15.0 \pm 1.5$ & $40.8 \pm 4.3$ \\
\hline
\end{tabular}

For compounds $\mathbf{1 1 a - i}$, the paralysis time of most compounds was less than 15 min, and the death time was more than 50 min, indicating that these compounds had significant paralysis effect on earthworms, but poor insecticidal action. In terms of shorter paralysis time $(3.6 \pm 0.2 \mathrm{~min})$ and death time $(8.1 \pm 0.8 \mathrm{~min})$, the anthelmintic effect of compound 11b was better than albendazole and our previous compounds, indicating that it has potential to be developed into a new anthelmintic drug [34]. For compounds 12a-h, the paralysis time was more than $15 \mathrm{~min}$ and death time was not discovered within $50 \mathrm{~min}$, indicating that these compounds had poor anthelmintic activity, even though they had good antibacterial activity.

\section{Materials and Methods}

\subsection{Chemical Reagents and Instruments}

Before using, tetrahydrofuran (THF) was dried with sodium under reflux, dichloromethane (DCM) was dried with $\mathrm{CaH}_{2}$ under reflux and triethylamine (TEA) was dried with molecular sieve. All the reagents above were chemically pure (Tianjin Tianli Chemical Reagent Co., Ltd., Tianjin, China). All the other reagents and compounds used in this study were commercially available analytically pure (AR) without further purification.

All the chemical reactions were detected by thin-layer chromatography (TLC), which was conducted on silica gel G plates (Taizhou Luqiao Sijia Biochemical Plastic Products Factory, Taizhou, China). The plates were observed under a Zf-2 tri-use ultraviolet analyzer (Shanghai Anting Electronic Instruments Factory, Shanghai, China). Most of the reaction mixtures were purified by column chromatography carried out on 200-300 mesh sieve specifications Silica Gel (Qingdao Ocean Chemical Co., Ltd., Qingdao, China).

All the purified compounds underwent the following tests to verify their purity and structure: a WRS-1A melting point apparatus (Shanghai Shinuo Physical Optical Instrument Co., Ltd., Shanghai, China) was used to assess the compounds' melting points. The ${ }^{1} \mathrm{H}-\mathrm{NMR}$ and ${ }^{13} \mathrm{C}-\mathrm{NMR}$ of each compound were recorded on an NMR spectrometer (Varian, Salt Lake City, UT, USA) in DMSO- $d_{6}$ with tetramethyl-silane (TMS) as an internal standard. The mass spectra (MS) of each compound were registered in an Agilent 1100 liquid chromatography-mass spectrometry (LC/MS) instrument (Agilent Technologies Co., Ltd., Santa Clara, CA, USA).

In addition, all the work in the biological activity test was completed in a DL-CJ-2N highperformance aseptic test stand (Beijing Donglian Haer Instrument Manufacturing Co., Ltd., Beijing, China). All biological media such as Mueller-Hinton (MH) broth medium, LuriaBertani (LB) broth medium and Nutrient Agar (Qingdao High-tech Park Haibo Biotech- 
nology Co., Ltd., Qingdao, China) were all dissolved directly with deionized water then sterilized by a vertical autoclave (Zealway Instrument Co., Ltd., Xiamen, China). Bacteria used in this experiment were grown in an MCO-20AIC carbon dioxide incubator (SANYO Co., Ltd., Osaka, Japan).

\subsection{Synthesis of the Target Compounds}

Intermediates $\mathbf{2 - 1 0}$ were synthesized according to the steps in the literature [31-34].

\subsubsection{General Procedure for the Preparation of Compounds (11a-e)}

Take compound (R)-(3-(6-morpholinopyridin-3-yl)-2-oxooxazolidin-5-yl) methyl acetate (11a) as an example. To a solution of compound $9(0.29 \mathrm{mmol})$, 4-dimethyl aminopyridine (DMAP, $0.06 \mathrm{mmol}$ ) and 1-ethyl-3-(3-dimethyl aminopropyl) carbodiimide (EDCI, $1.5 \mathrm{mmol})$ in $4 \mathrm{~mL}$ DCM, was added drop of acetyl chloride $(0.38 \mathrm{mmol})$ and stirred overnight. After the reaction completed, the reaction mixture was extracted with water and DCM $(5 \mathrm{~mL} \times 3)$. The combined organic phase was dried with anhydrous $\mathrm{Na}_{2} \mathrm{SO}_{4}$ and concentrated under reduced pressure. The target compound (11a) was separated by column chromatography (PE/EA, 3/1). Yield 72\%. A white solid, m. p. 92.9-95.1 ${ }^{\circ} \mathrm{C} ;{ }^{1} \mathrm{H}$ NMR $\left(600 \mathrm{MHz}, \mathrm{DMSO}-d_{6}\right) \delta 8.23\left(\mathrm{~d}, J=3.0 \mathrm{~Hz}, 1 \mathrm{H}, \mathrm{H}_{2}\right.$-pyridine), 7.83 (dd, $J=9.0,3.0 \mathrm{~Hz}, 1 \mathrm{H}$, $\mathrm{H}_{4}$-pyridine), 6.89 (d, $\mathrm{J}=9.0 \mathrm{~Hz}, 1 \mathrm{H}, \mathrm{H}_{5}$-pyridine), 4.94-4.90 (m, 1H, $\mathrm{H}_{5}$-oxazolidone), 4.31-4.24 (m, 2H, OCH $\mathrm{OCH}_{2}, 4.12-4.10$ (m, 1H, $\mathrm{H}_{4}$-oxazolidone), 3.80-3.77 (m, 1H, $\mathrm{H}_{4}$-oxazolidone), 3.72-3.68 (m, 4H, morpholine), 3.42-3.37 (m, 4H, morpholine), $2.06\left(\mathrm{~s}, 3 \mathrm{H}, \mathrm{CH}_{3}\right) ;{ }^{13} \mathrm{C} \mathrm{NMR}(150 \mathrm{MHz}$, DMSO- $\left.d_{6}\right) \delta 170.7,156.8,154.9,139.2,130.0,126.8,107.5,71.1,66.4,64.8,47.0,46.0,21.0$; ES-MS: $m / z$ calcd. for $\mathrm{C}_{15} \mathrm{H}_{19} \mathrm{~N}_{3} \mathrm{O}_{5}[\mathrm{M}+\mathrm{H}]^{+}: 322.1$; found: 322.110 .

(R)-(3-(6-morpholinopyridin-3-yl)-2-oxooxazolidin-5-yl)methyl cyclohexanecarboxylate (11b).

A pink solid; yield 65\%; m. p. 96.6-99.1 ${ }^{\circ} \mathrm{C} ;{ }^{1} \mathrm{H}$ NMR $\left(600 \mathrm{MHz}, \mathrm{DMSO}-d_{6}\right) \delta 8.24(\mathrm{~d}$, $J=3.0 \mathrm{~Hz}, 1 \mathrm{H}, \mathrm{H}_{2}$-pyridine), $7.83\left(\mathrm{dd}, J=9.0,3.0 \mathrm{~Hz}, 1 \mathrm{H}, \mathrm{H}_{4}\right.$-pyridine), 6.90 (d, $J=9.0 \mathrm{~Hz}$, $1 \mathrm{H}, \mathrm{H}_{5}$-pyridine), 5.03-4.96 (m, $1 \mathrm{H}, \mathrm{H}_{5}$-oxazolidone), 4.23-4.18 (m, 1H, $\left.\mathrm{OCH}_{2}\right), 4.04-3.92$ (m, $2 \mathrm{H}, \mathrm{OCH}_{2}$ and $\mathrm{H}_{4}$-oxazolidone), 3.76-3.74 (m, $1 \mathrm{H}, \mathrm{H}_{4}$-oxazolidone), 3.72-3.68 (m, $4 \mathrm{H}$, morpholine), 3.42-3.38 (m, 4H, morpholine), 3.09-3.05 (m, 4H, cyclohexane), 1.20-1.18 (m, $7 \mathrm{H}$, cyclohexane); ${ }^{13} \mathrm{C}$ NMR $\left(150 \mathrm{MHz}, \mathrm{DMSO}-d_{6}\right) \delta 175.8,159.3,154.4,146.4,135.1,114.8$, $106.7,78.9,65.8,44.8,43.8,41.5,28.6,25.3,25.2,14.1$; ES-MS: $m / z$ calcd. for $\mathrm{C}_{20} \mathrm{H}_{27} \mathrm{~N}_{3} \mathrm{O}_{5}$ $[\mathrm{M}+\mathrm{H}]^{+}:$390.2; found: 390.204 .

((R)-3-(6-morpholinopyridin-3-yl)-2-oxooxazolidin-5-yl)methyl tetrahydrofuran-2-carboxylate (11c).

Red oil; yield 51\%; ${ }^{1} \mathrm{H}$ NMR (600 MHz, DMSO- $\left.d_{6}\right) \delta 8.23\left(\mathrm{~d}, J=3.0 \mathrm{~Hz}, 1 \mathrm{H}, \mathrm{H}_{2}\right.$-pyridine), 7.83 (dd, $J=9.0,3.0 \mathrm{~Hz}, 1 \mathrm{H}, \mathrm{H}_{4}$-pyridine), 6.90 (d, $J=9.0 \mathrm{~Hz}, 1 \mathrm{H}, \mathrm{H}_{5}$-pyridine), 4.97-4.93 (m, $1 \mathrm{H}, \mathrm{H}_{5}$-oxazolidone), 4.48-4.27 (m, 3H, tetrahydrofuran), 4.15-4.12 (m, $\left.1 \mathrm{H}, \mathrm{OCH}_{2}\right)$, 3.82-3.73 (m, 3H, $\mathrm{OCH}_{2}$ and $\mathrm{H}_{4}$-oxazolidone), 3.73-3.66 (m, 4H, morpholine), 3.44-3.38 (m, $4 \mathrm{H}$, morpholine), 2.17-2.11 ( $\mathrm{m}, 1 \mathrm{H}$, tetrahydrofuran), 1.93-1.70 (m, 3H, tetrahydrofuran); ${ }^{13} \mathrm{C}$ NMR $\left(150 \mathrm{MHz}, \mathrm{DMSO}-d_{6}\right) \delta 173.1,159.4,154.4,146.4,135.1,114.7,106.7,78.5,77.6$, $68.5,65.8,44.8,41.4,37.2,30.0,24.8$; ES-MS: $m / z$ calcd. for $\mathrm{C}_{18} \mathrm{H}_{23} \mathrm{~N}_{3} \mathrm{O}_{6}[\mathrm{M}+\mathrm{H}]^{+}$: 378.2; found: 378.138 .

(R)-(3-(6-morpholinopyridin-3-yl)-2-oxooxazolidin-5-yl)methyl benzoate (11d).

A red solid; yield 62\%; m. p. $97.4-100.0{ }^{\circ} \mathrm{C} ;{ }^{1} \mathrm{H}$ NMR $\left(600 \mathrm{MHz}, \mathrm{DMSO}-d_{6}\right) \delta 8.24(\mathrm{~d}$, $J=3.0 \mathrm{~Hz}, 1 \mathrm{H}, \mathrm{H}_{2}$-pyridine), 7.83 (dd, $J=9.0,3.0 \mathrm{~Hz}, 1 \mathrm{H}, \mathrm{H}_{4}$-pyridine), 7.30-7.12 (m, 5H, benzene), 6.89 (d, $J=9.0 \mathrm{~Hz}, 1 \mathrm{H}, \mathrm{H}_{5}$-pyridine), 4.92-4.89 (m, 1H, $\mathrm{H}_{5}$-oxazolidone), 4.35-4.24 ( $\left.\mathrm{m}, 2 \mathrm{H}, \mathrm{OCH}_{2}\right), 4.13-4.08$ (m, 1H, $\mathrm{H}_{4}$-oxazolidone), 3.79-3.72 (m, 1H, $\mathrm{H}_{4}$-oxazolidone), 3.72-3.67 (m, 4H, morpholine), 3.45-3.34 (m, 4H, morpholine), 2.85-2.82 (m, 2H, $\left.\mathrm{CH}_{2}\right)$, 2.68-2.64 (m, 2H, CH 2$\left.) ;{ }^{13} \mathrm{C} \mathrm{NMR} \mathrm{(150} \mathrm{MHz,} \mathrm{DMSO-d} 6\right) \delta 171.9,159.4,154.5,146.4,141.2$, 135.2, 128.2, 128.1, 125.8, 114.8, 106.7, 78.9, 65.9, 44.8, 42.0, 41.6, 36.8, 31.1; ES-MS: $\mathrm{m} / \mathrm{z}$ calcd. for $\mathrm{C}_{22} \mathrm{H}_{25} \mathrm{~N}_{3} \mathrm{O}_{5}[\mathrm{M}+\mathrm{H}]^{+}$: 412.2; found: 412.148 .

(R)-(3-(6-morpholinopyridin-3-yl)-2-oxooxazolidin-5-yl)methyl nicotinate (11e).

A red solid; yield 65\%; m. p. $120.3-121.0{ }^{\circ} \mathrm{C} ;{ }^{1} \mathrm{H}$ NMR $\left(600 \mathrm{MHz}, \mathrm{DMSO}-d_{6}\right) \delta 9.05$ (d, $J=1.8 \mathrm{~Hz}, 1 \mathrm{H}$, pyridine), 8.82 (dd, $J=4.8,1.8 \mathrm{~Hz}, 1 \mathrm{H}$, pyridine), 8.29-8.23 (m, 2H, pyridine (B ring) and pyridine), $7.84\left(\mathrm{dd}, J=9.0,3.0 \mathrm{~Hz}, 1 \mathrm{H}, \mathrm{H}_{4}\right.$-pyridine (B ring)), 7.57 (dd, 
$J=7.8,4.8 \mathrm{~Hz}, 1 \mathrm{H}$, pyridine), $6.90\left(\mathrm{~d}, J=9.0 \mathrm{~Hz}, 1 \mathrm{H}, \mathrm{H}_{5}\right.$-pyridine (B ring $\left.)\right), 5.13-5.06(\mathrm{~m}, 1 \mathrm{H}$, $\mathrm{H}_{5}$-oxazolidone), 4.66-4.53 (m, $\left.2 \mathrm{H}, \mathrm{OCH}_{2}\right), 4.23-4.22$ ( $\mathrm{m}, 1 \mathrm{H}, \mathrm{H}_{4}$-oxazolidone), 4.01-3.99 (m, $1 \mathrm{H}, \mathrm{H}_{4}$-oxazolidone), 3.72-3.68 (m, $4 \mathrm{H}$, morpholine), 3.42-3.38 (m, $4 \mathrm{H}$, morpholine); ${ }^{13} \mathrm{C}$ NMR $\left(150 \mathrm{MHz}\right.$, DMSO- $\left.d_{6}\right) \delta 165.3,159.4,154.5,151.9,148.4,146.5,135.2,135.0,129.7$, 123.4, 114.7, 106.7, 78.5, 65.9, 44.8, 42.6, 37.5; ES-MS: $m / z$ calcd. for $\mathrm{C}_{19} \mathrm{H}_{20} \mathrm{~N}_{4} \mathrm{O}_{5}[\mathrm{M}+\mathrm{H}]^{+}$: 385.1; found: 385.133 .

Raw data for the above products are presented in Supplementary Materials (Figures S1-S16).

\subsubsection{General Procedure for the Preparation of Compounds (11f-i and 12a-h)}

Take compound (R)-(3-(6-morpholinopyridin-3-yl)-2-oxooxazolidin-5-yl) methyl cyclohexylcarbamate (11f) as an example. To a solution of compound $9(0.29 \mathrm{mmol})$, TEA $(0.58 \mathrm{mmol})$ in $4 \mathrm{~mL} \mathrm{DCM}$ was added drop of isocyanatocyclohexane $(0.44 \mathrm{mmol})$ under $0{ }^{\circ} \mathrm{C}$, then the mixture was raised to room temperature and stirred overnight. After the reaction completed, the mixture was extracted with water and DCM $(5 \mathrm{~mL} \times 3)$. The combined organic phase was dried with anhydrous $\mathrm{Na}_{2} \mathrm{SO}_{4}$ and concentrated under reduced pressure. The mixture was recrystallized with petroleum ether (PE) and ethyl acetate (EA) to obtain compound (11f). Yield 65\%. A red solid, m. p. 200.7-203.0 ${ }^{\circ} \mathrm{C} ;{ }^{1} \mathrm{H} \mathrm{NMR}(600 \mathrm{MHz}$, DMSO- $\left.d_{6}\right) \delta 8.24\left(\mathrm{~d}, J=3.0 \mathrm{~Hz}, 1 \mathrm{H}, \mathrm{H}_{2}\right.$-pyridine), $7.83\left(\mathrm{dd}, J=9.0,3.0 \mathrm{~Hz}, 1 \mathrm{H}, \mathrm{H}_{4}\right.$-pyridine), $7.27(\mathrm{~d}, J=7.8 \mathrm{~Hz}, 1 \mathrm{H}, \mathrm{NHCO}), 6.89\left(\mathrm{~d}, J=9.0 \mathrm{~Hz}, 1 \mathrm{H}, \mathrm{H}_{5}\right.$-pyridine), $4.89-4.85(\mathrm{~m}, 1 \mathrm{H}$, $\mathrm{H}_{5}$-oxazolidone), 4.28-4.16 (m, 2H, $\left.\mathrm{OCH}_{2}\right), 4.11-4.08\left(\mathrm{~m}, 1 \mathrm{H}, \mathrm{H}_{4}\right.$-oxazolidone), 3.86-3.72 ( $\mathrm{m}, 1 \mathrm{H}, \mathrm{H}_{4}$-oxazolidone), 3.71-3.69 (m, 4H, morpholine), 3.40-3.39 (m, 4H, morpholine), 1.75-1.59 (m, 4H, cyclohexane), 1.55-1.49 (m, 2H, cyclohexane), 1.29-1.20 (m, 3H, cyclohexane), 1.18-1.06 (m, 1H, cyclohexane), 1.04-0.96 (m, 1H, cyclohexane); ${ }^{13} \mathrm{C}$ NMR (150 MHz, DMSO- $\left.d_{6}\right) \delta 159.3,157.4,154.6,146.4,135.2,114.8,106.7,79.7,65.8,47.6,44.8,42.4,36.9,33.2$, 25.3, 24.4; ES-MS: $m / z$ calcd. for $\mathrm{C}_{20} \mathrm{H}_{28} \mathrm{~N}_{4} \mathrm{O}_{5}[\mathrm{M}+\mathrm{H}]^{+}:$405.2; found: 405.174 .

(R)-(3-(6-morpholinopyridin-3-yl)-2-oxooxazolidin-5-yl)methyl (4-chlorophenyl)carbamate (11g).

A red solid; yield $58 \%$; m. p. $185.0-187.9{ }^{\circ} \mathrm{C} ;{ }^{1} \mathrm{H}$ NMR $\left(600 \mathrm{MHz}\right.$, DMSO- $\left.d_{6}\right) \delta$ $8.90(\mathrm{~s}, 1 \mathrm{H}, \mathrm{NHCO}), 8.25\left(\mathrm{~d}, J=3.0 \mathrm{~Hz}, 1 \mathrm{H}, \mathrm{H}_{2}\right.$-pyridine), $7.84(\mathrm{dd}, J=9.0,3.0 \mathrm{~Hz}, 1 \mathrm{H}$, $\mathrm{H}_{4}$-pyridine), 7.50-7.46 (m, 2H, benzene), 7.35-7.31 (m, 2H, benzene), $6.89(\mathrm{~d}, J=9.0 \mathrm{~Hz}$, $1 \mathrm{H}, \mathrm{H}_{5}$-pyridine), 4.99-4.96 (m, 1H, $\mathrm{H}_{5}$-oxazolidone), 4.12-4.35 (m, 2H, $\left.\mathrm{OCH}_{2}\right), 4.15-4.12$ ( $\mathrm{m}, 1 \mathrm{H}, \mathrm{H}_{4}$-oxazolidone), 3.85-3.82 (m, $1 \mathrm{H}, \mathrm{H}_{4}$-oxazolidone), 3.71-3.69 $(\mathrm{m}, 4 \mathrm{H}$, morpholine), 3.43-3.38 ( $\mathrm{m}, 4 \mathrm{H}$, morpholine); ${ }^{13} \mathrm{C}$ NMR (150 MHz, DMSO- $\left.d_{6}\right) \delta 156.7,154.9,153.5,152.8$, $139.1,129.9,129.1,126.8,126.0,120.3,107.5,71.5,66.4,65.1,46.8,46.0$; ES-MS: $m / z$ calcd. for $\mathrm{C}_{20} \mathrm{H}_{21} \mathrm{ClN}_{4} \mathrm{O}_{5}[\mathrm{M}+\mathrm{H}]^{+}: 433.1$; found: 433.098 .

(R)-(3-(6-morpholinpyridin-3-yl)-2-oxooxazolidin-5-yl)methyl methanesulfonate (11h).

A yellow solid; yield $75 \%$; m. p. $184.2-185.9{ }^{\circ} \mathrm{C} ;{ }^{1} \mathrm{H}$ NMR $\left(600 \mathrm{MHz}\right.$, DMSO- $\left.d_{6}\right)$, $\delta: 8.23\left(\mathrm{~d}, J=3.0 \mathrm{~Hz}, 1 \mathrm{H}, \mathrm{H}_{2}\right.$-pyridine), $7.82\left(\mathrm{dd}, J=9.0,3.0 \mathrm{~Hz}, 1 \mathrm{H}, \mathrm{H}_{4}\right.$-pyridine), 6.90 (d, $J=9.0 \mathrm{~Hz}, 1 \mathrm{H}, \mathrm{H}_{5}$-pyridine), 5.02-4.96 (m, $1 \mathrm{H}, \mathrm{H}_{5}$-oxazolidone), 4.53-4.44 $(\mathrm{m}, 2 \mathrm{H}$, $\left.\mathrm{OCH}_{2}\right), 4.17-4.12\left(\mathrm{~m}, 1 \mathrm{H}, \mathrm{H}_{4}\right.$-oxazolidone), 3.80-3.76 (m, 1H, $\mathrm{H}_{4}$-oxazolidone), 3.71- 3.68 (m, 4H, morpholine), 3.41-3.39 (m, 4H, morpholine), $3.26\left(\mathrm{~s}, 3 \mathrm{H}, \mathrm{CH}_{3}\right) ;{ }^{13} \mathrm{C}$ NMR $(150 \mathrm{MHz}$, DMSO-d $\left.d_{6}\right) \delta 156.8,154.7,139.2,130.1,126.6,107.5,70.8,70.3,66.4,46.6,45.9,37.3$; ES-MS: $\mathrm{m} / z$ calcd. for $\mathrm{C}_{14} \mathrm{H}_{19} \mathrm{~N}_{3} \mathrm{O}_{6} \mathrm{~S}[\mathrm{M}+\mathrm{H}]^{+}$: 358.1; found: 358.096 .

(R)-(3-(6-morpholinpyridin-3-yl)-2-oxooxazolidin-5-yl)methyl benzenesulfonate (11i).

Red oil; yield $55 \%$; ${ }^{1} \mathrm{H} \mathrm{NMR}\left(600 \mathrm{MHz}\right.$, DMSO- $\left.d_{6}\right) \delta 8.13\left(\mathrm{~d}, J=3.0 \mathrm{~Hz}, 1 \mathrm{H}, \mathrm{H}_{2}\right.$-pyridine), $7.94\left(\mathrm{dd}, J=9.0,3.0 \mathrm{~Hz}, 1 \mathrm{H}, \mathrm{H}_{4}\right.$-pyridine), 7.85-7.80 (m, 1H, benzene), 7.63-7.59 (m, 2H, benzene), 7.36-7.26 (m, 2H, benzene), $7.16\left(\mathrm{~d}, J=9.0 \mathrm{~Hz}, 1 \mathrm{H}, \mathrm{H}_{5}\right.$-pyridine), $5.00-4.95(\mathrm{~m}$, $1 \mathrm{H}, \mathrm{H}_{5}$-oxazolidone), 4.41-4.33 (m, 1H, $\left.\mathrm{OCH}_{2}\right), 4.19-4.11\left(\mathrm{~m}, 1 \mathrm{H}, \mathrm{OCH}_{2}\right), 3.83-3.79(\mathrm{~m}, 2 \mathrm{H}$, $\mathrm{H}_{4}$-oxazolidone), 3.74-3.72 (m, $4 \mathrm{H}$, morpholine), 3.51-3.48 (m, $4 \mathrm{H}$, morpholine); ${ }^{13} \mathrm{C}$ NMR $\left(150 \mathrm{MHz}, \mathrm{DMSO}-d_{6}\right) \delta 159.9,154.9,146.9,141.0,135.7,132.9,129.7,126.9,115.1,107.2,79.1$, 66.3, 46.1 45.2, 37.6; ES-MS: $m / z$ calcd. for $\mathrm{C}_{19} \mathrm{H}_{21} \mathrm{~N}_{3} \mathrm{O}_{6} \mathrm{~S}[\mathrm{M}+\mathrm{H}]^{+}: 420.2$; found: 420.111 .

(R)-(3-(6-(4-(cyclohexanecarbonyl)piperazin-1-yl)pyridin-3-yl)-2-oxooxazolidin-5-yl)methyl methanesulfonate (12a).

A white solid; yield $73 \%$; m. p. $111.3-112.5{ }^{\circ} \mathrm{C} ;{ }^{1} \mathrm{H}$ NMR $\left(600 \mathrm{MHz}\right.$, DMSO- $\left.d_{6}\right) \delta$ $8.23\left(\mathrm{~d}, J=3.0 \mathrm{~Hz}, 1 \mathrm{H}, \mathrm{H}_{2}\right.$-pyridine), $7.84\left(\mathrm{dd}, J=9.0,3.0 \mathrm{~Hz}, 1 \mathrm{H}, \mathrm{H}_{4}\right.$-pyridine), 6.94 
(d, $J=9.0 \mathrm{~Hz}, 1 \mathrm{H}, \mathrm{H}_{5}$-pyridine), 5.02-4.98 (m, 1H, $\mathrm{H}_{5}$-oxazolidone), 4.54-4.44 (m, 2H, $\mathrm{OCH}_{2}$ ), 4.15-4.13 (m, $1 \mathrm{H}, \mathrm{H}_{4}$-oxazolidone), 3.82-3.73 (m, $1 \mathrm{H}, \mathrm{H}_{4}$-oxazolidone), 3.61-3.59 $\left(\mathrm{m}, 4 \mathrm{H}\right.$, piperazine), $3.50-3.48\left(\mathrm{~m}, 4 \mathrm{H}\right.$, piperazine), $3.26\left(\mathrm{~s}, 3 \mathrm{H}, \mathrm{CH}_{3}\right), 1.77-1.65(\mathrm{~m}, 11 \mathrm{H}$, cyclohexane); ${ }^{13} \mathrm{C}$ NMR (150 MHz, DMSO- $\left.d_{6}\right) \delta 174.1,154.7,139.2,130.3,128.9,126.5,107.9$, 70.8, 70.3, 46.6, 46.0, 45.4, 44.8, 37.3, 29.6, 26.1, 25.6; ES-MS: $m / z$ calcd. for $\mathrm{C}_{21} \mathrm{H}_{30} \mathrm{~N}_{4} \mathrm{O}_{6} \mathrm{~S}$ $[\mathrm{M}+\mathrm{H}]^{+}:$467.5; found: 467.183 .

(R)-(2-oxo-3-(6-(4-(4-(trifluoromethyl)benzoyl)piperazin-1-yl)pyridin-3-yl)oxazolidin-5-yl)methyl methanesulfonate (12b).

A white solid; yield $72 \%$; m. p. $164.2-165.3{ }^{\circ} \mathrm{C} ;{ }^{1} \mathrm{H}$ NMR (600 MHz, DMSO- $\left.d_{6}\right) \delta$ $8.24\left(\mathrm{~d}, J=3.0 \mathrm{~Hz}, 1 \mathrm{H}, \mathrm{H}_{2}\right.$-pyridine), 7.87-7.82 (m, 3H, $\mathrm{H}_{4}$-pyridine and benzene), 7.68 (d, $J=7.8 \mathrm{~Hz}, 2 \mathrm{H}$, benzene), $6.94\left(\mathrm{~d}, J=9.0 \mathrm{~Hz}, 1 \mathrm{H}, \mathrm{H}_{5}\right.$-pyridine $), 5.02-4.98(\mathrm{~m}, 1 \mathrm{H}$, $\mathrm{H}_{5}$-oxazolidone), $4.54-4.44\left(\mathrm{~m}, 2 \mathrm{H}, \mathrm{OCH}_{2}\right), 4.17-4.13\left(\mathrm{~m}, 1 \mathrm{H}, \mathrm{H}_{4}\right.$-oxazolidone), 3.80-3.60 (m, $5 \mathrm{H}, \mathrm{H}_{4}$-oxazolidone and piperazine), $3.49-3.36\left(\mathrm{~m}, 4 \mathrm{H}\right.$, piperazine), $3.26\left(\mathrm{~s}, 3 \mathrm{H}, \mathrm{CH}_{3}\right)$; ${ }^{13} \mathrm{C}$ NMR $\left(150 \mathrm{MHz}, \mathrm{DMSO}-d_{6}\right) \delta 168.3,166.4,156.3(\mathrm{~d}, J=231.1 \mathrm{~Hz}), 154.8,140.5,139.3$, 130.2, 128.3, 126.6, 126.0, 107.8, 70.8, 70.3, 46.6, 41.7, 40.5, 37.3.; ES-MS: $\mathrm{m} / \mathrm{z}$ calcd. for $\mathrm{C}_{22} \mathrm{H}_{23} \mathrm{~F}_{3} \mathrm{~N}_{4} \mathrm{O}_{6} \mathrm{~S}[\mathrm{M}+\mathrm{H}]^{+}$: 529.5; found: 529.104 .

(R)-(2-oxo-3-(6-(4-(3-phenylpropanoyl)piperazin-1-yl)pyridin-3-yl)oxazolidin-5-yl)methyl methanesulfonate (12c).

A yellow solid; yield $74 \%$; m. p. $133.0-135.0{ }^{\circ} \mathrm{C} ;{ }^{1} \mathrm{H}$ NMR (600 MHz, DMSO- $\left.d_{6}\right) \delta 8.22$ (d, $J=3.0 \mathrm{~Hz}, 1 \mathrm{H}, \mathrm{H}_{2}$-pyridine), $7.82\left(\mathrm{dd}, J=9.0,3.0 \mathrm{~Hz}, 1 \mathrm{H}, \mathrm{H}_{4}\right.$-pyridine), $7.31-7.24(\mathrm{~m}, 5 \mathrm{H}$, benzene), 6.91 (d, $J=9.0 \mathrm{~Hz}, 1 \mathrm{H}, \mathrm{H}_{5}$-pyridine), 5.014 .97 ( $\mathrm{m}, 1 \mathrm{H}, \mathrm{H}_{5}$-oxazolidone), 4.54-4.44 (m, 2H, $\left.\mathrm{OCH}_{2}\right), 4.16-4.13$ (m, 1H, $\mathrm{H}_{4}$-oxazolidone), 3.79-3.77 (m, 1H, $\mathrm{H}_{4}$-oxazolidone), 3.58-3.51 (m, $4 \mathrm{H}$, piperazine), 3.43-3.40 (m, $4 \mathrm{H}$, piperazine), $3.26\left(\mathrm{~s}, 3 \mathrm{H}, \mathrm{CH}_{3}\right), 2.86-2.76$ $\left(\mathrm{m}, 2 \mathrm{H}, \mathrm{CH}_{2}\right), 2.70-2.62\left(\mathrm{~m}, 2 \mathrm{H}, \mathrm{CH}_{2}\right) \cdot ;{ }^{13} \mathrm{C}$ NMR $\left(150 \mathrm{MHz}, \mathrm{DMSO}-d_{6}\right) \delta 170.6,156.3,154.7$, 141.9, 139.4, 130.2, 128.9, 128.7, 126.4, 126.3, 107.7, 70.8, 46.6, 45.6, 44.9, 41.1, 37.3, 34.5, 31.2; ES-MS: $m / z$ calcd. for $\mathrm{C}_{23} \mathrm{H}_{28} \mathrm{~N}_{4} \mathrm{O}_{6} \mathrm{~S}[\mathrm{M}+\mathrm{H}]^{+}: 489.56$; found: 489.163 .

$(R, E)-(2-o x o-3-(6-(4-(3-(p y r i d i n-3-y l) a c r y l o y l)$ piperazin-1-yl)pyridin-3-yl)oxazolidin-5-yl)methyl methanesulfonate (12d).

A yellow solid; yield $75 \%$; m. p. $104.5-106.7^{\circ} \mathrm{C} ;{ }^{1} \mathrm{H}$ NMR (600 MHz, DMSO- $\left.d_{6}\right) \delta 8.91$ (d, $J=2.4 \mathrm{~Hz}, 1 \mathrm{H}$, pyridine), $8.56(\mathrm{dd}, J=4.8,1.8 \mathrm{~Hz}, 1 \mathrm{H}$, pyridine), $8.25(\mathrm{~d}, J=3.0 \mathrm{~Hz}, 1 \mathrm{H}$, $\mathrm{H}_{2}$-pyridine (B ring)), 8.23-8.20 (m, $1 \mathrm{H}$, pyridine), 7.88-7.83 ( $\mathrm{m}, 1 \mathrm{H}, \mathrm{H}_{4}$-pyridine (B ring)), 7.58-7.46 (m, 3H, pyridine and alkene), 6.98-6.95 (m, 1H, $\mathrm{H}_{5}$-pyridine (B ring)), 5.02-4.79 (m, $1 \mathrm{H}, \mathrm{H}_{5}$-oxazolidone), 4.54-4.46 (m, 1H, $\left.\mathrm{OCH}_{2}\right), 4.19-4.05\left(\mathrm{~m}, 1 \mathrm{H}, \mathrm{OCH}_{2}\right), 3.88-3.66(\mathrm{~m}$, $6 \mathrm{H}, \mathrm{H}_{4}$-oxazolidone and piperazine), $3.56-3.51\left(\mathrm{~m}, 4 \mathrm{H}\right.$, piperazine), $3.27\left(\mathrm{~s}, 3 \mathrm{H}, \mathrm{CH}_{3}\right) .{ }^{13} \mathrm{C}$ NMR (150 MHz, DMSO- $\left.d_{6}\right) \delta 164.7,156.1,155.1,150.6,150.0,138.8,135.1,131.4,130.2,130.0$, $126.9,124.3,120.8,107.8,73.0,72.2,70.8,59.2,46.9,37.3$; ES-MS: $m / z$ calcd. for $\mathrm{C}_{22} \mathrm{H}_{25} \mathrm{~N}_{5} \mathrm{O}_{6} \mathrm{~S}$ $[\mathrm{M}+\mathrm{H}]^{+}$: 488.5; found: 489.181 .

(R)-(3-(6-(4-(cyclohexylcarbamoyl)piperazin-1-yl)pyridin-3-yl)-2-oxooxazolidin-5-yl)methyl methanesulfonate (12e).

A white solid; yield $61 \%$; m. p. $195.0-197.6{ }^{\circ} \mathrm{C} ;{ }^{1} \mathrm{H}$ NMR $\left(600 \mathrm{MHz}, \mathrm{DMSO}-d_{6}\right) \delta$ $8.22\left(\mathrm{~d}, J=3.0 \mathrm{~Hz}, 1 \mathrm{H}, \mathrm{H}_{2}\right.$-pyridine), $7.82\left(\mathrm{dd}, J=9.0,3.0 \mathrm{~Hz}, 1 \mathrm{H}, \mathrm{H}_{4}\right.$-pyridine), 6.93 $\left(\mathrm{d}, J=9.0 \mathrm{~Hz}, 1 \mathrm{H}, \mathrm{H}_{5}\right.$-pyridine), $6.25(\mathrm{~d}, J=7.8 \mathrm{~Hz}, 1 \mathrm{H}, \mathrm{NHCON}), 5.02-4.97(\mathrm{~m}, 1 \mathrm{H}$, $\mathrm{H}_{5}$-oxazolidone), 4.54-4.43 (m, $\left.2 \mathrm{H}, \mathrm{OCH}_{2}\right), 4.18-4.13\left(\mathrm{~m}, 1 \mathrm{H}, \mathrm{H}_{4}\right.$-oxazolidone), 3.80-3.77 (m, $1 \mathrm{H}, \mathrm{H}_{4}$-oxazolidone), $3.46-3.37\left(\mathrm{~m}, 8 \mathrm{H}\right.$, piperazine), $3.27\left(\mathrm{~s}, 3 \mathrm{H}, \mathrm{CH}_{3}\right), 1.78-1.73(\mathrm{~m}, 2 \mathrm{H}$, cyclohexane), 1.71-1.67 (m, 2H, cyclohexane), 1.60-1.55 (m, 1H, cyclohexane), 1.29-1.13 (m, $5 \mathrm{H}$, cyclohexane), $1.12-1.03$ (m, $1 \mathrm{H}$, cyclohexane); ${ }^{13} \mathrm{C}$ NMR (150 MHz, DMSO- $\left.d_{6}\right) \delta 157.3$, 156.6, 154.7, 139.4, 130.1, 126.3, 107.7, 70.8, 70.3, 49.6, 46.6, 45.4, 43.5, 37.3, 33.6, 25.9, 25.6; ES-MS: $m / z$ calcd. for $\mathrm{C}_{21} \mathrm{H}_{31} \mathrm{~N}_{5} \mathrm{O}_{6} \mathrm{~S}[\mathrm{M}+\mathrm{H}]^{+}: 482.2$; found: 482.203 .

(R)-((3-(6-(4-(4-chlorophenyl)carbamoyl)piperazin-1-yl)pyridin-3-yl)-2-oxooxazolidin-5-yl)methyl methanesulfonate (12f).

A white solid; yield $53 \%$; m. p. $210.5-212.9{ }^{\circ} \mathrm{C} ;{ }^{1} \mathrm{H}$ NMR $\left(600 \mathrm{MHz}, \mathrm{DMSO}-d_{6}\right) \delta$ $8.73(\mathrm{~s}, 1 \mathrm{H}, \mathrm{NHCON}), 8.24\left(\mathrm{~d}, J=3.0 \mathrm{~Hz}, 1 \mathrm{H}, \mathrm{H}_{2}\right.$-pyridine), $7.84(\mathrm{dd}, J=9.0,3.0 \mathrm{~Hz}, 1 \mathrm{H}$, $\mathrm{H}_{4}$-pyridine), 7.55-7.50 (m, 2H, benzene), 7.31-7.27 (m, 2H, benzene), $6.97(\mathrm{~d}, J=9.0 \mathrm{~Hz}$, $1 \mathrm{H}, \mathrm{H}_{5}$-pyridine), 5.03-4.98 (m, 1H, $\mathrm{H}_{5}$-oxazolidone), 4.53-4.46 (m, 2H, $\left.\mathrm{OCH}_{2}\right), 4.17-4.14$ 
(m, 1H, $\mathrm{H}_{4}$-oxazolidone), 3.81-3.78 (m, 1H, $\mathrm{H}_{4}$-oxazolidone), 3.58-3.56 (m, 4H, piperazine), 3.53-3.51 (m, 4H, piperazine), $3.27\left(\mathrm{~s}, 3 \mathrm{H}, \mathrm{CH}_{3}\right) ;{ }^{13} \mathrm{C} \mathrm{NMR}\left(150 \mathrm{MHz}, \mathrm{DMSO}-d_{6}\right) \delta 156.5$, 155.3, 154.7, 140.0, 139.4, 130.2, 128.7, 126.4, 125.8, 121.5, 107.8, 70.8, 70.3, 46.6, 45.4, 43.8, 37.3; ES-MS: $m / z$ calcd. for $\mathrm{C}_{21} \mathrm{H}_{24} \mathrm{ClN}_{5} \mathrm{O}_{6} \mathrm{~S}[\mathrm{M}+\mathrm{H}]^{+}: 510.1$; found: 510.122 .

(R)-(3-(6-(4-((4-fluorophenyl)sulfonyl)piperazin-1-yl)pyridin-3-yl)-2-oxooxazolidin-5-yl)methyl methanesulfonate $\mathbf{( 1 2 g )}$.

A white solid; yield 58\%; m. p. $215.7-218.3{ }^{\circ} \mathrm{C} ;{ }^{1} \mathrm{H}$ NMR $\left(600 \mathrm{MHz}, \mathrm{DMSO}-d_{6}\right) \delta 8.19$ (d, $J=3.0 \mathrm{~Hz}, 1 \mathrm{H}, \mathrm{H}_{2}$-pyridine), 7.87-7.82 (m, 2H, benzene), 7.81 (dd, $J=9.0,3.0 \mathrm{~Hz}, 1 \mathrm{H}$, $\mathrm{H}_{4}$-pyridine), 7.53-7.46 (m, 2H, benzene), 6.90 (d, $J=9.0 \mathrm{~Hz}, 1 \mathrm{H}, \mathrm{H}_{5}$-pyridine), 5.00-4.96 (m, 1H, $\mathrm{H}_{5}$-oxazolidone), 4.52-4.42 (m, 2H, $\left.\mathrm{OCH}_{2}\right), 4.14-4.11$ (m, 1H, $\mathrm{H}_{4}$-oxazolidone), 3.77-3.75 (m, 1H, $\mathrm{H}_{4}$-oxazolidone), 3.60-3.55 (m, 4H, piperazine), $3.26\left(\mathrm{~s}, 3 \mathrm{H}, \mathrm{CH}_{3}\right), 3.00-2.98$ (m, 4H, piperazine); $\left.{ }^{13} \mathrm{C} \mathrm{NMR} \mathrm{(150} \mathrm{MHz,} \mathrm{DMSO-d}\right)_{6} \delta 165.2(\mathrm{~d}, J=252.2 \mathrm{~Hz}), 155.7,154.7$, $138.9,131.2,130.2,126.8,117.3,108.1,70.8,70.3,46.5,45.9,44.9,37.3 ;$ ES-MS: $m / z$ calcd. for $\mathrm{C}_{20} \mathrm{H}_{23} \mathrm{FN}_{4} \mathrm{O}_{7} \mathrm{~S}_{2}[\mathrm{M}+\mathrm{H}]^{+}:$515.1; found: 515.107 .

(R)-(3-(6-(4-((2,4-difluorophenyl)sulfonyl)piperazin-1-yl)pyridin-3-yl)-2-oxooxazolidin-5-yl)methyl methanesulfonate $\mathbf{( 1 2 h )}$.

A white solid; yield 71\%; m. p. $214.6-215.6{ }^{\circ} \mathrm{C} ;{ }^{1} \mathrm{H}$ NMR $\left(600 \mathrm{MHz}, \mathrm{DMSO}-d_{6}\right) \delta 8.20$ (d, $J=3.0 \mathrm{~Hz}, 1 \mathrm{H}, \mathrm{H}_{2}$-pyridine), 7.82 (dd, $J=9.0,3.0 \mathrm{~Hz}, 1 \mathrm{H}, \mathrm{H}_{4}$-pyridine), 7.72-7.67 (m, $1 \mathrm{H}$, benzene), 7.11 ( $\mathrm{dd}, J=12.6,2.4 \mathrm{~Hz}, 1 \mathrm{H}$, benzene), 6.98 (dd, $J=9.0,2.4 \mathrm{~Hz}, 1 \mathrm{H}$, benzene), $6.91\left(\mathrm{~d}, J=9.0 \mathrm{~Hz}, 1 \mathrm{H}, \mathrm{H}_{5}\right.$-pyridine), 5.01-4.96 (m, 1H, $\mathrm{H}_{5}$-oxazolidone), 4.52-4.41 (m, 2H, $\left.\mathrm{OCH}_{2}\right)$, 4.15-4.11 (m, 1H, H4 -oxazolidone), 3.82-3.68 (m, 1H, $\mathrm{H}_{4}$-oxazolidone), 3.58-3.56 (m, $4 \mathrm{H}$, piperazine), $3.26\left(\mathrm{~s}, 3 \mathrm{H}, \mathrm{CH}_{3}\right), 3.10-3.07\left(\mathrm{~m}, 4 \mathrm{H}\right.$, piperazine). ${ }^{13} \mathrm{C} \mathrm{NMR}(150 \mathrm{MHz}$, DMSO- $\left.d_{6}\right) \delta 165.3,160.2\left(\mathrm{~d}, J_{C-F}=253.1 \mathrm{~Hz}\right), 155.9,154.7(\mathrm{~d}, J=176.7 \mathrm{~Hz}), 139.1,132.8$, $130.1,126.7,115.4,111.5,108.1,103.8,70.8,70.3,56.8,46.5,45.6,45.1 ;$ ES-MS: $m / z$ calcd. for $\mathrm{C}_{20} \mathrm{H}_{22} \mathrm{~F}_{2} \mathrm{~N}_{4} \mathrm{O}_{7} \mathrm{~S}_{2}[\mathrm{M}+\mathrm{H}]^{+}:$533.5; found: 533.206 .

Raw data for the above products are presented in Supplementary Materials (Figure S17-S52).

\subsection{Antibacterial Activity Assay}

3.3.1. Minimum Inhibitory Concentration (MIC) Study

According to the standard procedure recommended by the Clinical Laboratory Standards Institute (CLSI) [37], the minimum inhibitory concentration (MIC) in vitro of all synthesized compounds for Gram-positive strains was evaluated on 96-well microplates by the broth microdilution technique to screen for antibacterial activities. In MuellerHinton (MH) sterile medium, a series of compound solutions was prepared by double-fold dilution method from 0.5 to $256 \mu \mathrm{g} / \mathrm{mL}$. After that, $100 \mu \mathrm{L}$ diluted standard inoculum $\left(1 \times 10^{6} \mathrm{CFU} / \mathrm{mL}\right)$ was added into the gradient solution. The 96 -well plates were cultured at $37^{\circ} \mathrm{C}$ for $16-18 \mathrm{~h}$, and the MIC of each well was considered to be the lowest concentration that could significantly inhibit the growth of bacteria. The untreated inoculum was used as a negative control and linezolid was used as a positive control [38].

\subsubsection{Bacterial Inhibitory Zone Test}

The bacterial inhibitory zone of $\mathbf{1 2} \mathbf{e}$ against S. aureus (ATCC 25923) was determined by pore diffusion method [39]. A total of $100 \mu \mathrm{L}$ of fresh culture was dispersed on sterile petri dishes containing nutrient AGAR media. The holes were made in the petri plates using $100 \mu \mathrm{L}$ micro pipette tips and injected with varied concentrations of $12 \mathbf{e}(160,320$, 640 , and $1600 \mu \mathrm{g} / \mathrm{mL}$ ) and $40 \mu \mathrm{g} / \mathrm{mL}$ of control (linezolid), following which the petri plates were kept overnight at $37^{\circ} \mathrm{C}$. After incubation, a band was formed around the holes by inhibiting biological growth.

\subsubsection{Growth Kinetic Study}

Based on the previous result of antibacterial activity, compound $12 \mathbf{e}$ was selected for further research. According to the literature [36], the growth kinetics of Bacillus subtilis (BNCC 109047) was determined after treatment with compound 12e and linezolid. The log-phase strain was diluted to $10^{6}-10^{7} \mathrm{CFU} / \mathrm{mL}$ in LB broth containing compound 
12e at a series of concentrations $(0,1 / 4,1 / 2,1$ and $2 \times \mathrm{MIC})$, then incubated at $37^{\circ} \mathrm{C}$ and $150 \mathrm{rpm}$. Linezolid at the concentration of $2 \mu \mathrm{g} / \mathrm{mL}$ was used as a positive control. At the beginning, culture mixture $(3 \mathrm{~mL})$ was removed from each sample solution at intervals of $1 \mathrm{~h}$, and turbidity was measured by ultraviolet spectrophotometer at $600 \mathrm{~nm}$. When the O.D. value became stable, the time interval was gradually increased and the time-kill curve was drawn.

\subsection{Binding Mode Study}

Molecular docking assay was performed using the AutoDock 4.2.6 ${ }^{\circledR}$ software. The X-ray crystal 3D structure of the 50S ribosomal subunit (PDB ID: 3CPW) was obtained from the Protein Data Bank (PDB) database. The ligands and proteins were removed from the structure and RNA chains were retained using PyMOL 1.5.0.3. The modified structure was prepared for further molecular docking. 2D structures of the ligands were built with the aid of ChemDraw 19.0 and the 3D structures were established by Chem3D 19.0 software. The docking results were analyzed and visualized with PyMOL 1.5.0.3.

\subsection{Anthelmintic Activity Assay}

Due to the high similarity between adult Indian earthworms ( $P$. posthuma) and intestinal roundworms (A. bricoides) in terms of anatomical and physiological characteristics [40], anthelmintic activities of all compounds were evaluated [41,42].

Before use, the earthworms with an average length of $6-10 \mathrm{~cm}$ and width of $0.2-0.3 \mathrm{~cm}$ were cleaned with normal saline solution [41], and the target compounds and positive control drug (albendazole) were dissolved with the minimum amount of DMSO and diluted with normal saline solution.

Then, the tested solution with a massive concentration of $2 \mathrm{~g} / \mathrm{L}$ was prepared and placed in a Petri dish $(\mathrm{d}=6 \mathrm{~cm})$. After being divided into different groups with six (6) worms, they were transferred to Petri dishes with sterile forceps and the paralysis and death time were monitored and recorded with stopwatches for $1 \mathrm{~h}$. Earthworms tested in standard saline $(0.9 \% w / v)$ were taken as the control group. The paralysis time was considered as the time when the worm showed no obvious movement at rest or under mild vibration. In a similar way, death time was considered when there was no obvious movement after severe shaking and exposure to $50{ }^{\circ} \mathrm{C}$ water $[41,42]$. The mean paralysis and death times were was used to reflect the anthelmintic effect of these compounds; the shorter time, the better the effect.

\section{Conclusions}

Seventeen novel 3-(3-pyridyl)-oxazolidone-5-methyl derivatives were designed and synthesized, and their structures were identified by ${ }^{1} \mathrm{H}$ NMR, ${ }^{13} \mathrm{C}$ NMR and MS. The primary screening of antibacterial activity, which was tested on Staphylococcus aureus (ATCC25923), Streptococcus pneumoniae (ATCC49619), Bacillus subtilis (BNCC109047) and Staphylococcus epidermidis (BNCC186652), showed that most of the compounds had certain antibacterial activity, among which compound 12a and 12e had the best antimicrobial activity. In a further bactericidal kinetic assay, 12e was mainly interfering in the logarithmic phase of bacteria in a concentration-dependent manner. Molecular docking between the 12e and the $50 \mathrm{~S}$ ribosomal subunit was studied to predict the mechanism of action. In addition, the anthelmintic activity test demonstrated that the compound $\mathbf{1 1} \mathbf{b}$ had the most significant anthelmintic effect. These results provide a preliminary basis for the development of novel pyridine heterocyclic compounds as antibacterial and anthelmintic agents. Further structural optimization and biological activity confirmation of the action model are in progress.

Supplementary Materials: Figure S1: Structure and Numbering of 11a, Figure S2: ${ }^{1} \mathrm{H}$ NMR Spectrum of 11a, Figure S3: ${ }^{13} \mathrm{C}$ NMR Spectrum of 11a. Figure S4: ES-MS for compound 11a, Figure S5: ${ }^{1} \mathrm{H}$ NMR Spectrum of 11b, Figure S6: ${ }^{13}$ C NMR Spectrum of 11b. Figure S7: ES-MS for compound 
11b, Figure S8: ${ }^{1} \mathrm{H}$ NMR Spectrum of 11c, Figure S9: ${ }^{13} \mathrm{C}$ NMR Spectrum of 11c. Figure S10: ESMS for compound 11c, Figure S11: ${ }^{1} \mathrm{H}$ NMR Spectrum of 11d, Figure S12: ${ }^{13} \mathrm{C}$ NMR Spectrum of 11d. Figure S13: ES-MS for compound 11d, Figure S14: ${ }^{1} \mathrm{H}$ NMR Spectrum of 11e, Figure S15: ${ }^{13} \mathrm{C}$ NMR Spectrum of 11e. Figure S16: ES-MS for compound 11e, Figure S17: ${ }^{1} \mathrm{H}$ NMR Spectrum of 11f, Figure S18: ${ }^{13} \mathrm{C}$ NMR Spectrum of 11f. Figure S19: ES-MS for compound 11f, Figure S20: ${ }^{1} \mathrm{H}$ NMR Spectrum of 11g, Figure S21: ${ }^{13} \mathrm{C}$ NMR Spectrum of 11g. Figure S22: ES-MS for compound 11g, Figure S23: ${ }^{1} \mathrm{H}$ NMR Spectrum of 11h, Figure S24: ${ }^{13} \mathrm{C}$ NMR Spectrum of $11 \mathrm{~h}$. Figure S25: ES-MS for compound 11h, Figure S26: ${ }^{1} \mathrm{H}$ NMR Spectrum of 11i, Figure S27: ${ }^{13} \mathrm{C}$ NMR Spectrum of 11i. Figure S28: ES-MS for compound 11i, Figure S29: ${ }^{1} \mathrm{H}$ NMR Spectrum of 12a, Figure S30: ${ }^{13} \mathrm{C}$ NMR Spectrum of 12a. Figure S31: ES-MS for compound 12a, Figure S32: ${ }^{1} \mathrm{H}$ NMR Spectrum of 12b, Figure S33: ${ }^{13} \mathrm{C}$ NMR Spectrum of 12b. Figure S34: ES-MS for compound 12b, Figure S35: ${ }^{1} \mathrm{H}$ NMR Spectrum of 12c, Figure S36: ${ }^{13} \mathrm{C}$ NMR Spectrum of 12c. Figure S37: ES-MS for compound 12c, Figure S38: ${ }^{1} \mathrm{H}$ NMR Spectrum of 12d, Figure S39: ${ }^{13} \mathrm{C}$ NMR Spectrum of 12d. Figure S40: ES-MS for compound 12d, Figure S41: ${ }^{1} \mathrm{H}$ NMR Spectrum of 12e, Figure S42: ${ }^{13} \mathrm{C}$ NMR Spectrum of 12e. Figure S43: ES-MS for compound 12e, Figure S44: ${ }^{1} \mathrm{H}$ NMR Spectrum of 12f, Figure S45: ${ }^{13} \mathrm{C}$ NMR Spectrum of 12f. Figure S46: ES-MS for compound 12f, Figure S47: ${ }^{1} \mathrm{H}$ NMR Spectrum of 12g, Figure S48: ${ }^{13} \mathrm{C}$ NMR Spectrum of 12g. Figure S49: ES-MS for compound 12g, Figure S50: ${ }^{1} \mathrm{H}$ NMR Spectrum of 12h, Figure S51: ${ }^{13} \mathrm{C}$ NMR Spectrum of 12h. Figure S52: ES-MS for compound 12h.

Author Contributions: Conceptualization, B.J. and H.-1.Y.; methodology, B.J., J.-y.C., Z.-1.S., M.-q.S. and H.-1.Y.; software, Z.-1.S.; validation, B.J., J.-y.C., Z.-1.S., M.-q.S. and H.-1.Y.; formal analysis, B.J. and H.-1.Y.; investigation, H.-1.Y.; resources, H.-1.Y.; data curation, B.J. and H.-1.Y.; writing-original draft preparation, B.J.; writing-review and editing, H.-1.Y.; visualization, Z.-1.S.; supervision, H.-1.Y.; project administration, H.-1.Y.; funding acquisition, H.-1.Y.. All authors have read and agreed to the published version of the manuscript.

Funding: This research was funded by the National Natural Science Foundation of China (Grant No. 31802227).

Institutional Review Board Statement: Not applicable.

Informed Consent Statement: Not applicable.

Data Availability Statement: Not applicable.

Conflicts of Interest: The authors declare no conflict of interest.

Sample Availability: Samples of the compounds are not available from the authors.

\section{References}

1. Chen, L.; Wang, X. Molecular mechanism of bacterial drug resistance based on embedded system and rapid detection method of drug resistance gene. Microprocess. Microsyst. 2021, 82, 103912. [CrossRef]

2. Lin, X.J.; Zou, J.; Yao, K.; Li, L.; Zhong, L. Analysis of antibiotic sensitivity and resistance genes of Bordetella pertussis in Chinese children. Medicine 2021, 100, e24090. [CrossRef] [PubMed]

3. Obp, A.; Jose, F. Bacterial upper respiratory tract infections in Brazil: Bacterial resistance, human resistance, scientific darkness. Braz. J. Otorhinolaryngol. 2021, 87, 123. [CrossRef]

4. Liu, C.H.; Xue, W.Q. Comparisons of Japanese Kanpo medicine and Chinese patent medicine for medication guidance. Int. Med. Health Guid. News 2019, 25, 585.

5. Denegre, A.A.; Myers, K.; Fefferman, N.H. Impact of Strain Competition on Bacterial Resistance in Immunocompromised Populations. Antibiotics 2020, 9, 114. [CrossRef]

6. Bassetti, M.; Poulakou, G.; Ruppe, E.; Bouza, E.; Van Hal, S.J.; Brink, A. Antimicrobial resistance in the next 30 years, humankind, bugs and drugs: A visionary approach. Intensive Care Med. 2017, 43, 1464-1475. [CrossRef]

7. Ding, Y.J.; Nie, Y.; Yu, Y. Anti-microbial Activities of Protic Ionic Liquids Studied with Microcalorimetry Method. Chem. Res. Chin. Univ. 2011, 4, 132.

8. $\quad$ Ding, H.H.; Zhao, M.H.; Zhai, L.; Zhen, J.B.; Yang, K.W. Quinine-based quaternized polymer: A potent scaffold with bactericidal properties without resistance. Polym. Chem. 2021, 12, 2397. [CrossRef]

9. Motsoeneng, T.S.; Mochane, M.J.; Mokhena, T.C.; Sadiku, E.R. Recent Progress on Antibiotic Polymer/Metal Nanocomposites for Health Applications. In Antibiotic Materials in Healthcare; Academic Press: Santiago, Chile, 2020. [CrossRef]

10. Zhang, Q.; Chu, X.; Buckling, A. Overcoming the growth-infectivity trade-off in a bacteriophage slows bacterial resistance evolution. Evol. Appl. 2021, 14, 7. [CrossRef] 
11. Zheng, W.; Jia, Y.; Zhao, Y.; Zhang, J.; Jiang, X. Reversing Bacterial Resistance to Gold Nanoparticles by Size Modulation. Nano Lett. 2021, 21, 5. [CrossRef]

12. Abouelhassan, Y.; Garrison, A.; Yang, H.; Riveros, A.C.; Burch, G.; Huigens, R.W. Recent Progress in Natural Product-Inspired Programs Aimed at Addressing Antibiotic Resistance and Tolerance. J. Med. Chem. 2019, 62, 7618. [CrossRef]

13. Valente, L.; Prazak, J.; Que, Y.A.; Cameron, D.R. Progress and Pitfalls of Bacteriophage Therapy in Critical Care: A Concise Definitive Review. Crit. Care Explor. 2021, 3, e0351. [CrossRef]

14. Zhao, Y.; Chen, L. Nanomaterial-based strategies in antimicrobial applications: Progress and perspectives. Nano Res. 2021, 14, 4417-4441. [CrossRef]

15. Shariati, A.; Dadashi, M.; Chegini, Z.; van Belkum, A.; Mirzaii, M.; Khoramrooz, S.S.; Darban-Sarokhalil, D. The global prevalence of Daptomycin, Tigecycline, Quinupristin/Dalfopristin, and Linezolid-resistant Staphylococcus aureus and coagulase-negative staphylococci strains: A systematic review and meta-analysis. Antimicrob. Resist. Infect. Control. 2020, 9, 56. [CrossRef]

16. Bao, P.; Zhang, Z.; Zhao, W.; Jiang, Y.; Wang, D. Isolation and whole genome sequencing of a new type of linezolid-resistant Enterococcus faecalis from two cases of infective endocarditis after renal transplantation. J. Glob. Antimicrob. Resist. 2020, 20, 346. [CrossRef]

17. Le, T.; Nguyen, K.; Le, T.; Nguyen, K.; Le, P.; Cafini, F.; Duong, H.X. The emergence of plasmid-borne cfr-mediated linezolid resistant-staphylococci in Vietnam. J. Glob. Antimicrob. Resist. 2020, 22, 462. [CrossRef]

18. Maarouf, L.; Omar, H.; El-Nakeeb, M.; Abouelfetouh, A. Prevalence and mechanisms of linezolid resistance among staphylococcal clinical isolates from Egypt. Eur. J. Clin. Microbiol. Infect. Dis. 2020, 40, 815. [CrossRef]

19. Christos, M.; Taha, A.A.; Shirin, J.; Charlotte, H.; Melanie, C.; Mark, S.; Khondaker, M.R. Synthesis, microbiological evaluation and structure activity relationship analysis of linezolid analogues with different C5-acylamino substituents. Bioorg. Med. Chem. 2021, 8, 116397. [CrossRef]

20. Xu, B.X.; Ding, X.D.; Tang, H.; Xiang, M.; Xu, B.; Li, Y.; Han, W. One-step Synthesis of N-Doped Mesoporous Carbon as Highly Efficient Support of Pd Catalyst for Hydrodechlorination of 2,4-Dichlorophenol. Chem. Res. Chin. Univ. 2018, 34, 51. [CrossRef]

21. Feng, F.; Jiang, L.; Yuting, G. Mitochondrion-anchoring AIEgen with Large Stokes Shift for Imaging-guided Photodynamic Therapy. Chem. Res. Chin. Univ. 2021, 37, 668. [CrossRef]

22. García-Olaiz, G.D.; Alcántar-Zavala, E.; Ochoa-Terán, A.; Cabrera, A.; Laniado-Laborín, R. Design, synthesis and evaluation of the antibacterial activity of new Linezolid dipeptide-type analogues. Bioorg. Chem. 2019, 95, 103483. [CrossRef]

23. Qureshi, S.I.; Chaudhari, H.K. Design, synthesis, in-silico studies and biological screening of Quinazolinone analogues as potential antibacterial agents against MRSA. Bioorg. Med. Chem. 2019, 27, 2676. [CrossRef]

24. Song, D.; Zhang, N.; Zhang, P.; Zhang, N.; Ma, S. Design, synthesis and evaluation of novel 9-arylalkyl-10-methylacridinium derivatives as highly potent FtsZ-targeting antibacterial agents. Eur. J. Med. Chem. 2021, 221, 113480. [CrossRef]

25. Xu, S.; Jiang, J.; Qi, Y.; Ding, X.; Zhao, Y. Design and synthesis of biaryloxazolidinone derivatives containing amide or acrylamide moiety as novel antibacterial agents against Gram-positive bacteria. Bioorg. Med. Chem. Lett. 2019, 29, 126747. [CrossRef]

26. Eleazar, A.Z.; Esteban, H.G.; Adrián, O.T.; Julio, M.Á.; Edgar, A.E.Z.; Alex, J.S.M.; Efraín, A.; Alberto, C.; Gerardo, A.; Valentín, M.S.; et al. Novel Linezolid analogues with antiparasitic activity against Hymenolepis nana. Bioorg. Chem. 2020, 105, 104359. [CrossRef]

27. Iqbal, M.A.; Husain, A.; Alam, O.; Khan, S.A.; Ahmad, A.; Haider, M.R.; Alam, M.A. Design, synthesis, and biological evaluation of imidazopyridine-linked thiazolidinone as potential anticancer agents. Arch. Pharm. 2020, 353, 2000071. [CrossRef]

28. Pankaja, K.K. Aminoalkylpyridine compounds which are useful as anitconvulsant drugs, excitatory amino acid inhibitors and NMDA sigma receptor antagonists. U.S. Patent 5648369, 15 July 1997.

29. Kuthyala, S.; Hanumanthappa, M.; Kumar, S.M.; Sheik, S.; Prabhu, A. Crystal, Hirshfeld, ADMET, drug-like and anticancer study of some newly synthesized imidazopyridine containing pyrazoline derivatives. J. Mol. Struct. 2019, 1197, 65. [CrossRef]

30. Ribeiro, J.; Soares, J.; Almeida, S.; Soares, J.; Alves, J.; Ribeiro, A.G.; Lima, M. COVID-19 therapy: What weapons do we bring into battle? Bioorganic Med. Chem. 2020, 29, 115855. [CrossRef]

31. Salituro, F.; Bemis, G.; Evindar, G. Pyridine Derivatives as Inhibitors of P38. Patent WO070695, 27 September 2001.

32. Yang, H.L.; Xu, G.X.; Pei, Y.Z. Synthesis, Preliminary Structure-activity Relationships and Biological Evaluation of Pyridinyl-4,5-2H-isoxazole Derivatives as Potent Antitumor Agents. Chem. Res. Chin. Univ. 2017, 33, 61. [CrossRef]

33. Jin, B.; Tao, Y.; Yang, H.L. Synthesis, Biological Evaluation and Molecular Docking of Novel Phenylpyrimidine Derivatives as Potential Anticancer Agents. Chem. Res. Chin. Univ. 2018, 34, 6. [CrossRef]

34. Yang, H.L.; Jin, B. Synthesis and Biological Activity of Pyridinyl-4,5-2H-isoxazole Heterocyclic Derivatives. Fine Chem. 2019, 36, 487. [CrossRef]

35. Nicholas, A. Synopsis of Some Recent Tactical Application of Bioisosteres in Drug Design. J. Med. Chem. 2011, 54, 2529. [CrossRef]

36. Aneja, B.; Azam, M.; Alam, S.; Perwez, A.; Maguire, R.; Yadava, U.; Kavanagh, K.; Daniliuc, C.G.; Rizvi, M.M.A.; Haq, Q.M.R.; et al. Natural Product-Based 1,2,3-Triazole/Sulfonate Analogues as Potential Chemotherapeutic Agents for Bacterial Infections. ACS Omega 2018, 3, 6912-6930. [CrossRef] [PubMed]

37. CLSI. M100 Performance Standards for Antimicrobial Susceptibility Testing, 29th ed.; CLSI: Wayne, PA, USA, 2019.

38. Zhang, L.; Fu, Y.H.; Ding, Y.; Meng, J.; Wang, Z.C.; Wang, P.Y. Antibacterial Activity of Novel 18 $\beta$-Glycyrrhetinic Hydrazide or Amide Derivatives. Chem. Res. Chin. Univ. 2021, 37, 662. [CrossRef] 
39. Gunasekaran, S.; Sivaji, S.; Sandhanasamy, D.; Mohamad, S.A.; Aruliah, R.; Murali, K.M.; Ranganathan, B. Phytosynthesis of silver nanoparticles from Jatropha integerrima Jacq. flower extract and their possible applications as antibacterial and antioxidant agent. Saudi J. Biol. Sci. 2021, in press. [CrossRef]

40. Niaz, A.; Syed, S.; Ismail, S.; Ghayour, A.; Mehreen, G.; Imran, K. Cytotoxic and anthelmintic potential of crude saponins isolated from Achillea Wilhelmsii, C. Koch and Teucrium Stocksianum boiss. BMC Complementary Altern. Med. 2011, 11, 106. [CrossRef]

41. Ayaz, M.; Junaid, M.; Subhan, F.; Ullah, F.; Sadiq, A.; Ahmad, S.; Imran, M.; Kamal, Z.; Hussain, S. Heavy metals analysis, phytochemical, phytotoxic and anthelmintic investigations of crude methanolic extract, subsequent fractions and crude saponins from Polygonum hydropiper L. BMC Complementary Altern. Med. 2014, 14, 465. [CrossRef]

42. Chitikina, S.S.; Buddiga, P.; Deb, P.K.; Mailavaram, R.P.; Kar, S. Synthesis and anthelmintic activity of some novel (E)-2-methyl/propyl-4-(2-(substitutedbenzylidene)hydrazinyl)-5,6,7,8-tetrahydrobenzo[4,5]thieno[2,3-d]pyrimidines. Med. Chem. Res. 2020, 29, 1600. [CrossRef] 